\title{
Attitude Control of Cubesat in Single Axis by Fuzzy Logic Controller
}

\author{
Sedat Nazlibilek ${ }^{1}$, Ozlem Gulsum Kilickaya ${ }^{2}$, Süleyman Gökhun Tanyer ${ }^{1}$ \\ ${ }^{1}$ Department of Electrical-Electronics Engineering, Faculty of Engineering, Baskent University \\ 06810, Ankara, Turkey \\ snazlibilek@baskent.edu.tr, gokhuntanyer@baskent.edu.tr \\ ${ }^{2}$ Atilim University \\ ozlemkilickaya@gmail.com
}

\begin{abstract}
Over the last two decades, satellites are getting smaller with increasing capabilities for space research. Small satellites (between 1 and $15 \mathrm{~kg}$ ) show great promise as a low-cost option both in production and launching. In this work, attitude control problem for a cubesat is examined. A physical testbed is designed for simulation and testing in a single axis. Cubesat reaction wheel and the testbed are integrated, and fuzzy logic control software in Arduino Mega microprocessor environment is developed. The cubesat floor at the same axis of reaction wheel is tested and real-time measurement data are obtained. Step response and ramp response behaviours are analysed.
\end{abstract}

Keywords: Attitude control, cubesat, cubesat missions, miniaturized satellites, reaction wheel.

\section{Introduction}

Today, satellites are getting smaller since size limitations provide better cost effective solutions both in production and launching. In this trend, miniature satellites are getting more favourable. A class of miniature satellites for space research, cubesats are first introduced by California Polytechnic State University and Stanford University by 1999. Cubesat has a volume of exactly one litre $(10 \mathrm{~cm} \times 10 \mathrm{~cm} \times 10 \mathrm{~cm})$, and its mass is approximately 1 kilogram which is called 1-Unit or $1 \mathrm{U}$. Its size can be enlarged as $2 \mathrm{U}, 3 \mathrm{U}$, etc. by combining several U's. Cubesats have various missions such as taking photo from space, earthquake detection, detection of cosmic dust, climatology purposes like prediction of rain and tornado, etc. Their position must be controlled due to their missions and other requirements in space. A cubesat's orbit cannot be changed in space however its attitude can be controlled using reaction wheels or any other actuators such as magnetotorquers or small thrusters [2, 3]. In satellite communications, the attitude control is the term used to describe the orientation of the satellite in relationship to Earth and Sun $[4,5]$. Its orientation may require some control actions despite of there is no move and change in placement of Cubesat. Miniaturized attitude control system for nanosatellites was discussed in [6]. In this work, attitude control problem for a cubesat in a single-axis is examined [7, 8]. Attitude Stabilization for the Nano Remote Sensing Satellite and another work by the same team namely three-axis attitude control by two-step rotations using only magnetic torquers were presented in $[9,10]$. Design and verification of operating procedures of the ADCS of a nanosatellite was discussed in [11]. A physical testbed composed of a plate representing one side of a cubesat is designed for simulation and testing in a single axis. To the authors best knowledge, this paper is the first application of the fuzzy controller for the problem of attitude control of a cubesat. The cubesat reaction wheel and the testbed are integrated, and real-time measurement data are obtained. Fuzzy logic control software is developed in Arduino Mega microprocessor environment [1]. Step and ramp responses are obtained and analysed.

\section{Simulation of Single-axis CubeSat Attitude Control System}

In this work, attitude control problem for a cubesat is examined, and a practical method is proposed. A physical testbed is designed for simulation and testing in a single axis. The reaction wheel and the testbed are integrated, and realtime measurement data are obtained. Results are illustrated using a testing environment where Matlab based fuzzy control software and an Arduino Mega is utilized. It is observed that this practical method could also be applied to three dimensional attitude control problems. The attitude behaviour of cubesat is investigated in the plane which the reaction 
wheel is coupled. The surface normal defines the axis of rotation of the reaction wheel. The torque generated can be in counter-clockwise

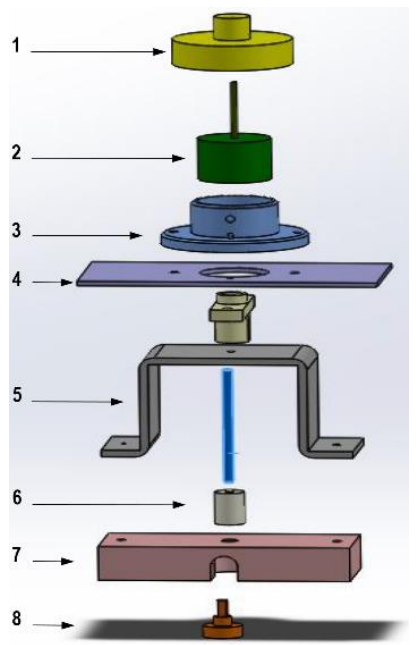

(a)

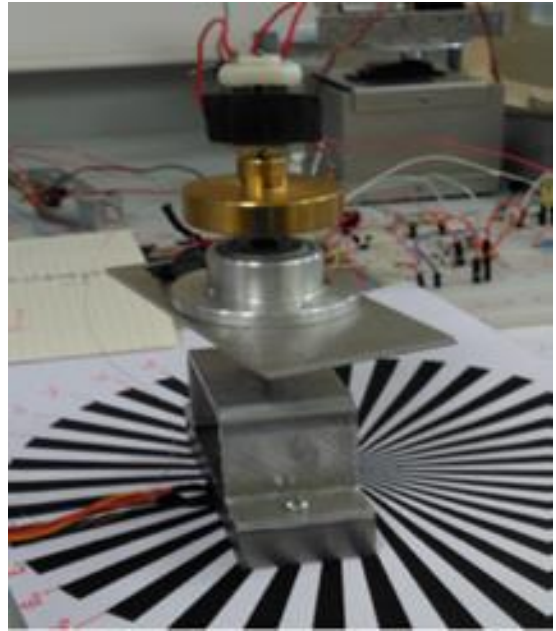

(b)

Fig. 1: Reaction wheel system used in measurements and tests. (a) The compact reaction wheel system is composed of (1) the brass reaction wheel, (2) the DC Motor (brushed), (3) the motor holder, (4) the plate that representing the Cubesat floor, (5) the plate support,

(6) the plate rotation shaft, (7) the bearing of the shaft, (8) the base, (9) the potentiometer for plate position feedback; (b) The photograph of the system.

(CCW) in the opposite direction of the reaction wheel's angular rotation. The "control" requirements on a single axis can simply be defined by bringing the orientation of the plane in a desired position, and further in the presence of external distribution, correcting the position of cubesat. The testing prototype system consists of a reaction wheel, and the plate as shown in Fig. 1. The attitude behaviour of the system is analysed in the following section.

\subsection{Behaviour of the System under Control}

Single plane represents one of the sides of cubesat. Reaction wheel's torque is aligned by this plane and the torque applied to cubesat can be determined by Newton's second law. The rotational kinetic energy can be described by

$$
E_{k}=\frac{1}{2} J \omega^{2}
$$

where $J$ represents the moment of inertia and $\omega$ represents the platform's angular rate, and the angular momentum is given by

$$
L=J \omega .
$$

The angular position and the velocity in single axis is determined by inertia due to torque generated by external disturbance and the reaction wheel. The following relations describe the behavior of the system.

$$
\begin{gathered}
J_{p} \dot{\omega}=T_{d}-T_{\mathrm{w}} \\
J_{\omega}\left(\dot{\omega}+\dot{\omega}_{w}\right)=T_{\mathrm{w}} \\
\dot{\omega}=\frac{T_{d}}{J_{p}}-\frac{T_{w}}{J_{p}}
\end{gathered}
$$




$$
\dot{\omega}_{w}=\frac{T_{w}}{J_{r}}-\frac{T_{d}}{J_{j p}}
$$

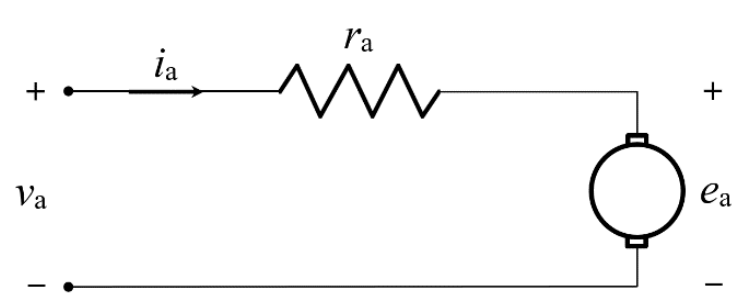

(a)

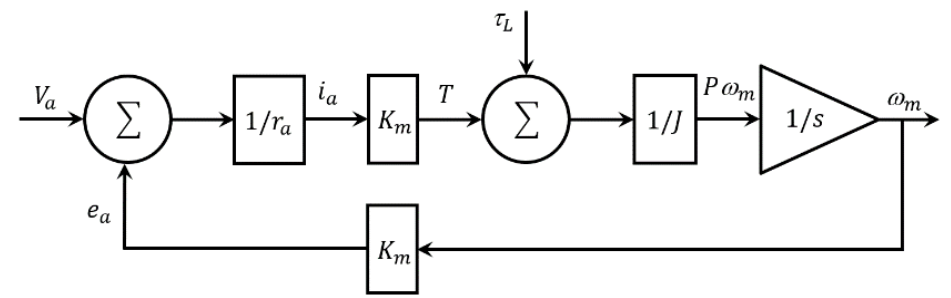

(b)

Fig. 2: (a) The equivalent circuit model of the dc motor. (b) The closed-loop model of the DC motor.

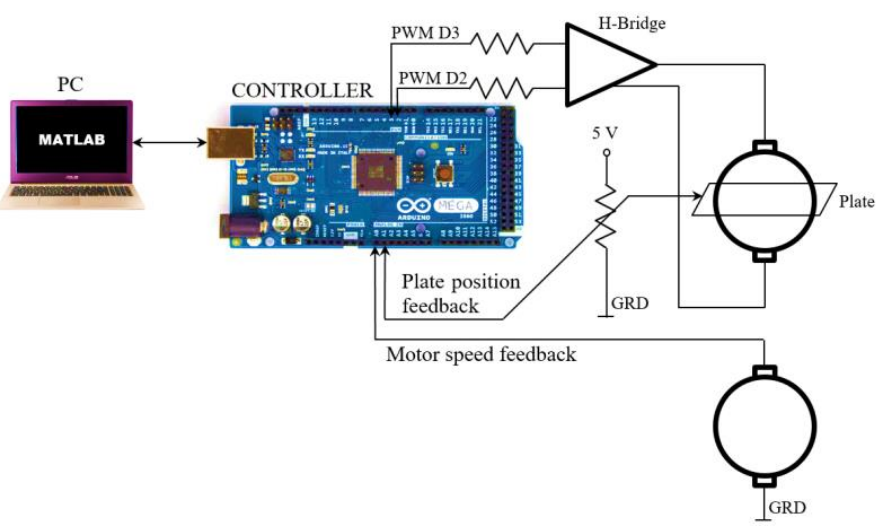

Fig. 3: The schematics of the overall control system.

where $J_{\mathrm{p}}$ represents the platform around the vertical axis, $T_{\mathrm{d}}$ represents the disturbance torque, $T_{\mathrm{w}}$ represents the torque of reaction wheel, $J_{\mathrm{w}}$, represents the inertia of the reaction wheel, and $\dot{\omega}_{\mathrm{w}}$ angular rate relative of wheel to plate. The mean inertia of cubesat can be defined by

$$
J_{r}=\frac{J_{p} J_{\mathrm{w}}}{J}
$$

This suggests that single axis angular position of cubesat can be controlled by the reaction wheel driven by an electric motor.

\subsection{Mathematical Model for DC Motor Coupled with Reaction Wheel}

The reaction wheel consists of a permanent magnet DC motor. The equivalent circuit for the DC motor is illustrated in Fig. 2 (a). The closed-loop model for the motor coupled with a load (reaction wheel) is illustrated in Fig. 2 (b). It is observed the input voltage causes the output speed, $\omega_{\mathrm{m}}$. The equivalent circuit given in Fig. 2 (a) suggests the following.

$$
\begin{gathered}
T=K_{m} i_{a} \\
e_{a}=K_{m} \omega_{m} \\
i_{a}=\left(V_{t a}-e_{a}\right) / r_{a}
\end{gathered}
$$

where $K_{\mathrm{m}}=K_{\mathrm{a}} \Phi_{\mathrm{d}}$ is the torque constant of the motor which is a function of motor geometry and the magnet properties, 
and the torque given in (8) is the mechanical torque. Thus, the torque of a permanent magnet motor is given by the product of the torque constant and the armature current.

\subsection{The Control System}

The overall system is shown in Fig. 3. The reaction wheel system is composed of reaction wheel, its driving motor and the testbed simulating cubesat floor. Two different system feedback channels are necessary to control the attitude of the plate which could be assumed to be the cubesat's floor. One of the feedbacks is the speed of DC motor, and the other is the position information about the plate orientation. The controller is implemented using Matlab, and also the integrated Arduino Mega

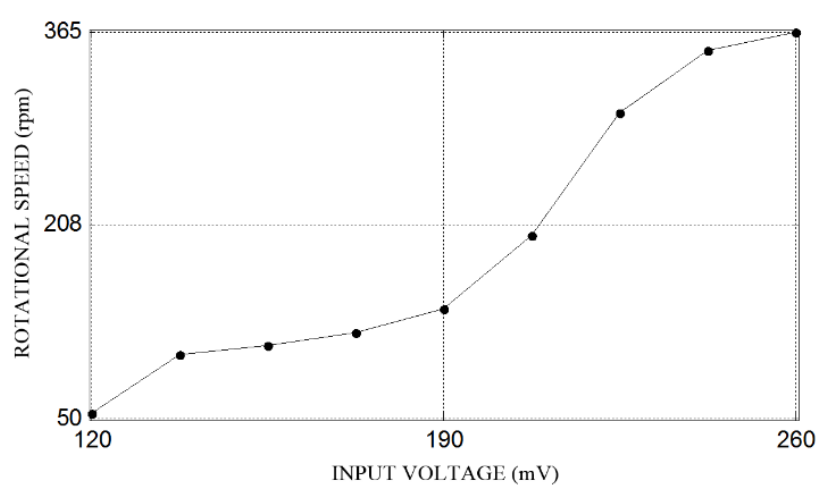

(a)

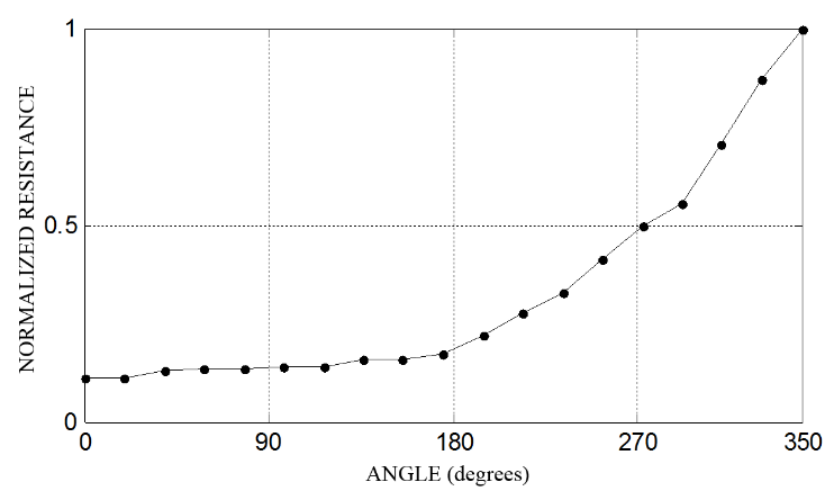

(b)

Fig. 4: (a) The voltage - speed characteristic of the DC generator is used for speed feedback. (b) The angle degree - resistance characteristic of the potentiometer is used for the position feedback.

routines. The controller is a fuzzy logic controller. Arduino Mega is the processor that implement receiving both feedback signals and transmitting pulse-width modulation (PWM) signal driving the motor in clockwise (CW) and counterclockwise $(\mathrm{CCW})$ directions. The position feedback of the plate is received by a potentiometer connected mechanically to the rotating shaft of the plate. The speed feedback of the motor is received by a DC generator connected mechanically to the center of the reaction wheel. The feedback signals are used for calculating the error, $e(t)$, and the derivative of the error, $d / d t(e(t))$. Those real-time measurements are compared with the desired values. The control signal is produced by the fuzzy controller. It is based on if-then Rules linguistically, and the output of the fuzzy controller is converted to numerical values. Then, they are converted to PWM signals. The motor is driven by these PWM signals which are transmitted by the Arduino processor. These signals are powered by the H-Bridge up to a level of driving the motor, and the brass reaction wheel with sufficient torque. The position and the velocity can simply be corrected by rotating the motor in appropriate direction. The motor speed can be decreased by the fuzzy controller around the desired angle value until the plate is located at the desired angular position. Here, the variables such as feedback signals and output PWM signals are measured both by the oscilloscope and multi-meter connected to the appropriate terminals. The signals are also displayed in the laptop by Matlab program, and results are given in the following section.

\subsection{The Control Method of the System}

The method of control is selected to be an intelligent control namely fuzzy logic control. The fuzzy logic control is preferred since exact analytical control model for the plant is not required. Fuzzy approach enables the control the system similar to the control by human using linguistic variables. The fuzzifier stage implements the input membership functions of error and error rate. The if-then rules are implemented based on the membership functions. The output is obtained from one of the appropriate if-then rules and converted into a numerical value by the defuzzifier. The linguistic variable obtained at the output (decrease/increase the speed) is then converted into a PWM signal with appropriated width. Fuzzy controller can be designed by dividing the control problem into several steps. At the initial step, desired command value and the controlled variable itself are determined. Then, error, $\mathrm{e}(\mathrm{t})$, and the time derivative of error are calculated by comparing the

desired command and the controlled variable. The adjectives of the error and derivative of error are determined. Next, the output adjectives are selected. These are necessary for setting of the rule matrix, input membership function and output 
membership function. If-then rules are determined using the rule matrix, adjectives of the error and derivative of error inputs. The final step is the defuzzification. An inference method is selected based on the specifications of the requirements, and the numerical value for the output is calculated.

\section{a. Inputs:}

In this work, the steps of fuzzy logic control may be described as in the following. The sensor outputs are initially set to the desired values. Desired level of command signal varies in the range of $0.0-5.0 \mathrm{~V}$. The DC generator feedback voltage varies between $0.0 \mathrm{mV}$ and $260 \mathrm{mV}$ as shown in Fig. 4 (a). This voltage provides the feedback for current speed. The potentiometer degree - resistance characteristic is given in Fig. 4 (b). This value is the the feedback for the position of the plate representing the floor of cubesat.

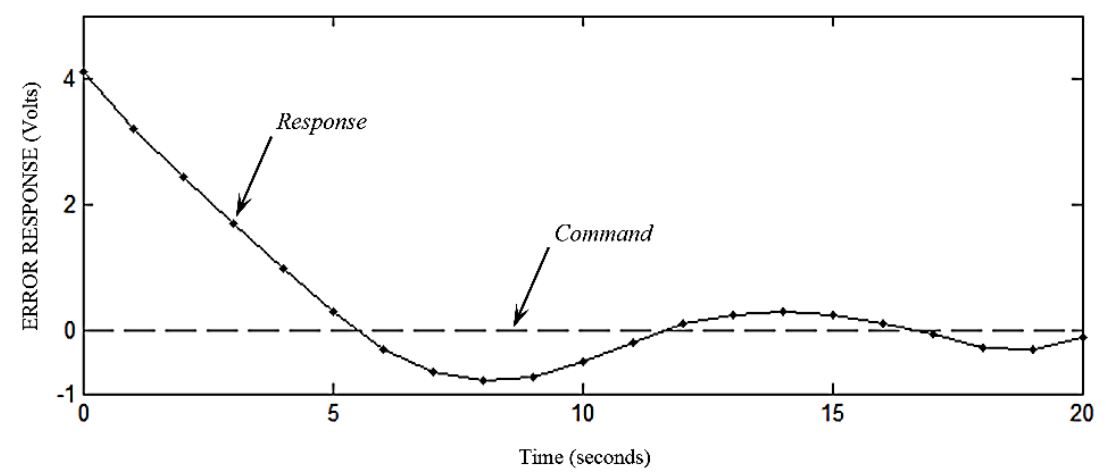

Fig. 5: The error response and the command signal for desired value.

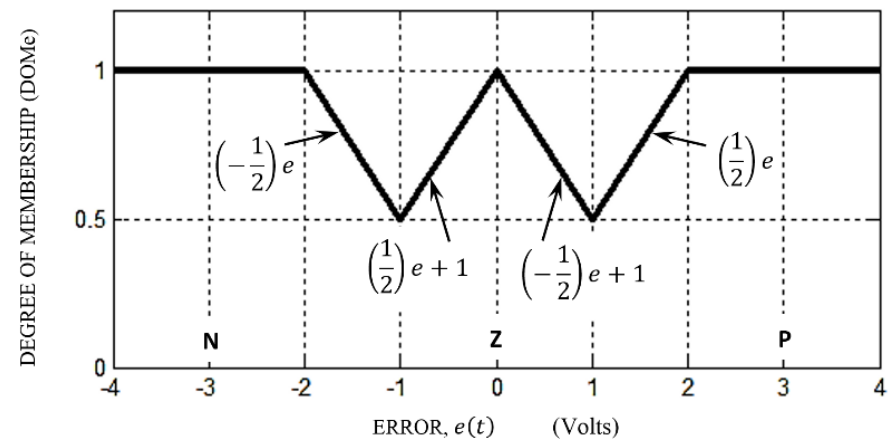

(a)

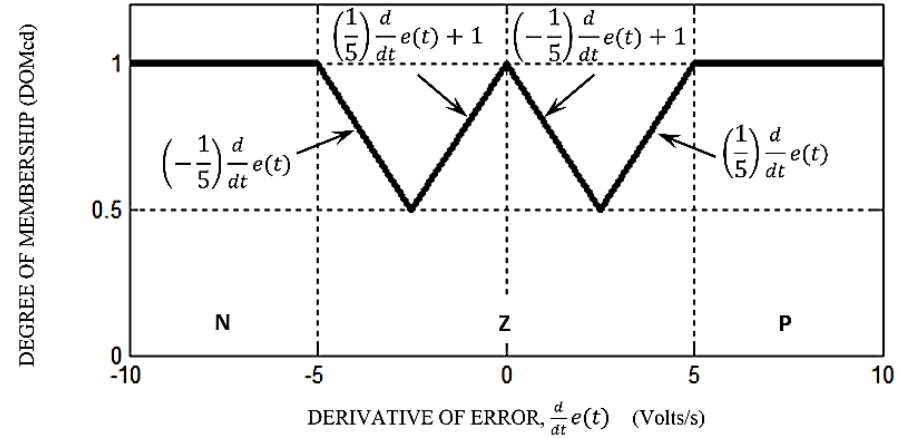

(b)

Fig. 6: Input membership functions.

The position feedback resistance is normalized to represent the general case. The voltage output of DC generator is multiplied by a feedback constant $K=20$ to fit it to 5 Volts. The error response and the command signal is illustrated in Fig. 5 for which the adjectives of error and its derivative are determined. Command signal is the target (desired) speed, $r(t)$, and the current speed is the feedback, $c(t)$, which is measured by the sensor. Then, the error, $e(t)$, can be defined by the difference.

$$
e(t)=r(t)-c(t)
$$

The linguistic variables for the fuzzy controller are defined as follows.
If $e(t)>0$
(Positive, $P$ ), it means that the motor is too slow.
If $e(t)<0$
(Negative, $N$ ), it means that the motor is too fast.
If $e(t)=0$
(Zero, $Z$ ), it means that the motor speed is just right. The change of error is $d / d t e(t)$.
If $d / d t e(t)>0$
(Positive, $P$ ), it means that the motor is getting faster.
If $d / d t e(t)<0$
(Negative, $N$ ), it means that the motor is getting slower. 
If $d / d t e(t)=0 \quad$ (Zero, Z), it means that zero change in error.

The output adjectives are "Fast, $F$ ", "No Change, $N C$ ", and "Slow, $S$ ". The set of inputs and the conclusion are summarized as follows.

Input \#1: ‘“Error”, $P, Z, N\}$

Input \#2: ‘“Change of Error”, $P, Z, N\}$

Conclusion: $\{“ O u t p u t ”, F, N C, S$ \}.

\section{b. Input membership functions:}

Input membership functions can be defined as illustrated in Fig. 6.

\begin{tabular}{|c|c|c|c|c|}
\hline \multirow[t]{3}{*}{$\begin{array}{l}\text { Derivative of error, } \\
\qquad \frac{d}{d t} e(t)\end{array}$} & $\mathbf{N}$ & $\underset{(\mathrm{R} 1)}{\mathbf{S}}$ & $\begin{array}{c}\mathbf{F} \\
(\mathrm{R} 2)\end{array}$ & $\underset{(\mathrm{R} 3)}{\mathbf{F}}$ \\
\hline & Z & $\underset{(\mathrm{R} 4)}{\mathbf{S}}$ & $\begin{array}{l}\mathbf{N C} \\
\text { (R5) }\end{array}$ & $\underset{(\mathrm{R} 6)}{\mathbf{F}}$ \\
\hline & $\mathbf{P}$ & $\underset{(\mathrm{R} 7)}{\mathbf{S}}$ & $\underset{(\mathrm{R} 8)}{\mathbf{S}}$ & $\underset{(\mathrm{R} 9)}{\mathbf{F}}$ \\
\hline
\end{tabular}

Fig. 7: The rule matrix.

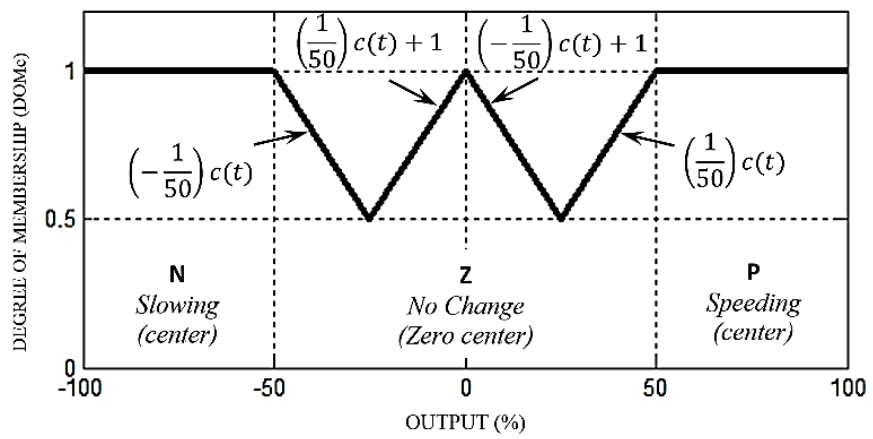

Fig. 8: The output membership function.

The membership functions are defined in three regions, namely Negative $(\mathrm{N})$. Zero $(\mathrm{Z})$, and Positive $(\mathrm{P})$. The resolution can be increased according to the desired response of the system, and the boundaries of the regions are determined by the operation ranges. The piece-wise linear regions are illustrated in Fig. 6 . The vertical axis is the degree of membership (DOM). The positive membership values are less than 1. DOMe represents the degree of membership of the error, and DOMed represents the degree of membership of derivative of the error. The horizontal axis of the error membership function is given in $\mathrm{rad} / \mathrm{sec}$. The horizontal axis of the membership function of the derivative of the error is given $\mathrm{rad} / \mathrm{sec} 2$. If a value of the error observed to fall down to any of the regions $(\mathrm{N}, \mathrm{Z}$, or $\mathrm{P})$, then the value of DOM is obtained by mapping this value to the vertical axis. The rule list and the rule matrix are given as follows.
If $(e<0)$ and $(e d<0)$
then $\quad$ Slow $=$ DOMe \& DOMed $=$ R1
If $(e=0)$ and $(e d<0) \quad$ then
Fast $=$ DOMe \& DOMed $=$ R2
If $(e>0)$ and $(e d<0)$
then $\quad$ Fast $=$ DOMe \& DOMed $=\mathrm{R} 3$
If $(e<0)$ and $(e d=0)$
then $\quad$ Slow $=$ DOMe \& DOMed $=\mathrm{R} 4$
If $(e=0)$ and $(e d=0)$
then $\quad$ No Change $=$ DOMe \& DOMed $=\mathrm{R} 5$
If $(e>0)$ and $(e d=0)$
then $\quad$ Fast $=$ DOMe \& DOMed $=\mathrm{R} 6$
If $(e<0)$ and $(e d>0) \quad$ then $\quad$ Slow $=$ DOMe \& DOMed $=\mathrm{R} 7$
If $(e=0)$ and $(e d>0)$ then Slow $=$ DOMe \& DOMed $=\mathrm{R} 8$ 
If $(e>0)$ and $(e d>0)$ then Slow $=$ DOMe \& DOMed $=\mathrm{R} 9$

where, error $e(t)$ is given in (13), and $e d$ is the derivative of error with respect to time. The operation (DOMe \& DOMed) is an AND operation such that the result is the minimum of the operands. $R j$, where $j=1,2 \ldots, 9$ is the consequence of the operation. It gets a value from the set $\{\mathrm{S}, \mathrm{NC}, \mathrm{F}\}$ meaning Slow, No Change, and Fast, respectively. The rule matrix for the operation is given in Fig. 7. The rule matrix defines the consequences based on the antecedents.

\section{c. Defuzzification and output generation:}

The last step in fuzzy logic control is the operation called defuzzification. This step converts linguistic values into numerical values. An inference method is selected for defuzzification. There are several inference methods such as "maxmin", "max-

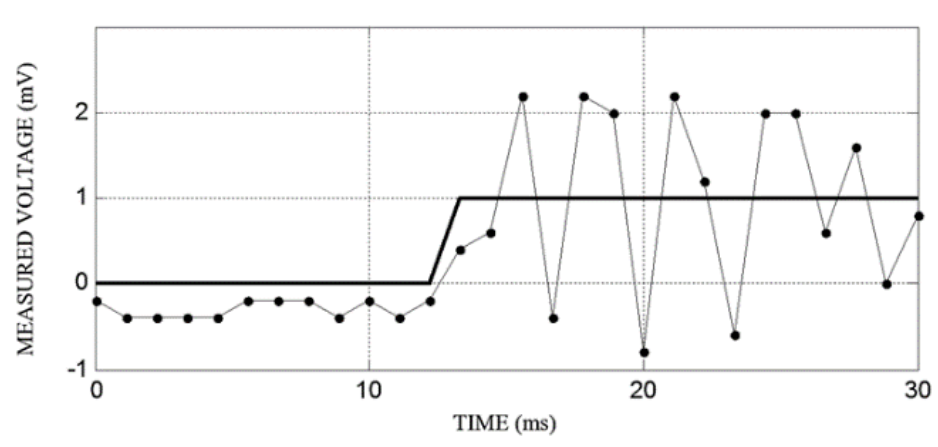

(a)



(b)

Fig. 9: The measured responses for; (a) step, (b) ramp inputs.

product", "averaging", and "root-sum-square" which are some of the most popular inference methods. In this work, rootsum-square (RSS) method is selected for inferencing. The proposed combination of rules is; scaling the inputs and calculating the strengths of the values of the "output membership function" as illustrated in (Fig. 8).

Three regions can be defined for the output membership function as illustrated in Fig. 8. Those regions are as follows; $\{\mathrm{S}, \mathrm{NC}, \mathrm{F}\}$, slowing (Negative), $\mathrm{Z}$ (No Change), and speeding (Positive), respectively. The strengths of each inference are calculated as follows.

$$
\begin{gathered}
\text { Neg } g_{\text {Strength }}=\left(R_{1}^{2}+R_{2}^{2}+R_{7}^{2}+R_{8}^{2}\right)^{1 / 2} \\
N o_{\text {Change }}=\left(R_{5}^{2}\right)^{1 / 2} \\
\text { Pos }_{\text {Strenght }}=\left(R_{2}^{2}+R_{3}^{2}+R_{6}^{2}+R_{9}^{2}\right)^{1 / 2} .
\end{gathered}
$$

The output value for the strengths is calculated by multiplying the center points of the region of the output membership function. Later, resulting value is divided by the sum of the center points using

$$
c=\frac{\left(N e g_{\text {Strength }} * N e g_{\text {Center }}+N o_{\text {Change }} * Z_{\text {ero }} o_{\text {Center }}+\text { Pos }_{\text {Strength }} * \text { Pos }_{\text {Center }}\right)}{\left(N e g_{\text {Strength }}+N o_{\text {Change }}+\text { Pos }_{\text {Strength }}\right)}
$$

where $c$ is the output providing the percentage strength measured at the horizontal axis of the membership function. It has a numerical value where it is projected to DOMc as an activation parameter (). This value is later mapped to the pulse-width modulation (PWM) output as the final step of the approach.

\section{Results and Conclusion}

Attitude control problem for a cubesat is examined, and a physical testbed is designed and manufactured for simulation and testing in a single axis. To the authors best knowledge, this paper is the first application of the fuzzy 
controller for the problem of attitude control of a cubesat. Results are illustrated using a testing environment where Matlab based fuzzy control software and an Arduino Mega is utilized. It is observed that this practical method could also be applied to three dimensional attitude control problems. The system is tested using two different test inputs, namely, step and ramp (Fig. 9). Step input represents sudden change in external forces where ramp input represents gradual changes. Various input signals and their immediate responses are measured by sensors. It is observed that the error for step response is limited to approximately 15 to 20 degrees, and similarly error for ramp function is found to be limited to approximately 3 to 5 degrees. Step input causes a fast and sudden movement in the output which is undesired in space. It is observed to increase error in positioning and lowering the control quality. This could be much worse in space due to the lack of smoothing friction forces. Step function input is found to require special care. Ramp function however, causes less error and better control quality, comparatively. Algorithm design and development studies in standard laboratory environment are expected to be affected by friction force limitations. A cubesat is normally free in vacuum moving on a specified orbit, however, the testbed is faced with physical friction forces. During the tests, it was observed that the responses have large fluctuations. The reason for it is that the resolution of membership functions is somehow coarse. There are only three regions in membership functions. If the number of regions is increased, then smoother responses can be achieved. However, results show that practical approaches and development of solutions are still possible. Another observation is that there is a steady-state error in the responses. The reason for it is that only proportional and derivative of error signal are used. To eliminate the steady-state error, it is better to use integral of the error additionally. In the future, the proposed method can be illustrated and tested in three dimensional scenarios.

\section{References}

[1] L. A. Zadeh, "Fuzzy Sets," Information and control, vol. 8, no. 3, pp. 338-353, 1965.

[2] M. Lovera, E. De Marchi, and S. Bittanti "Periodic attitude control techniques for small satellites with magnetic actuators," IEEE Trans. on Control Systems Technology, vol. 10, no. 1, pp. 90-95, 2002.

[3] J. R. Forbes and C. J. Damaren, "Geometric approach to spacecraft attitude control using magnetic and mechanical actuation," Journal of Guidance, Control, and Dynamics, vol. 33, no. 2, pp. 590-595, 2010.

[4] J. Kim, and K. Worrall, "Sun tracking controller for UKube-1 using magnetic torquer only," IFAC Proceedings, vol. 46, no. 19, 541-546, 2013.

[5] N. Sugimura, T. Kuwahara, and K. Yoshida, "Attitude determination and control system for nadir pointing using magnetorquer and magnetometer," IEEE Aerospace Conference, pp. 1-12, 2016.

[6] G. P. Candini, F. Piergentili, and F. Santoni, "Miniaturized attitude control system for nanosatellites," Acta Astronautica, vol. 81, no. 1, pp. 325-334, 2012.

[7] S. O. Karpenko, M. Y. Ovchinnikov, D. S. Roldugin, and S. S. Tkachev, "One-axis attitude of arbitrary satellite using magnetorquers only," Cosmic Research, vol. 51, no. 6, pp. 478-484, 2013.

[8] M. Y. Ovchinnikov, D. S. Roldugin, S. S. Tkachev, and S. O. Karpenko, "New one-axis one-sensor magnetic attitude control theoretical and in-flight performance," Acta Astronautica, vol. 105, no. 1, pp. 12-16, 2014.

[9] T. Inamori, K. Shimizu, Y. Mikawa, T. Tanaka, and S. Nakasuka, "Attitude Stabilization for the Nano Remote Sensing Satellite PRISM,” Journal of Aerospace Engineering, vol. 26, no. 3, pp. 594-602, 2013.

[10] T. Inamori, K. Otsuki, Y. Sugawara, P. Saisutjarit, and S. Nakasuka, "Three-axis attitude control by two-step rotations using only magnetic torquers in a low Earth orbit near the magnetic equator," Acta Astronautica, vol. 128, pp. 696-706.

[11] T.-Y. Lin, and J.-C. Juang, "Design and verification of the operating procedure of attitude determination and control subsystem of a nanosatellite," CACS International Automatic Control Conference (CACS 2014), pp. 5156, 2014. 